\title{
Performance of Polymeric Skin Adhesives during Perspiration
}

Hansen, Daniel; Moghaddam, Saeed Zajforoushan; Eiler, Johannes; Hansen, Kristoffer; Thormann, Esben

\section{Published in:}

ACS Applied Polymer Materials

Link to article, DOI:

10.1021/acsapm.9b01214

Publication date:

2020

Document Version

Publisher's PDF, also known as Version of record

Link back to DTU Orbit

Citation (APA):

Hansen, D., Moghaddam, S. Z., Eiler, J., Hansen, K., \& Thormann, E. (2020). Performance of Polymeric Skin Adhesives during Perspiration. ACS Applied Polymer Materials, 2(4), 1535-1542.

https://doi.org/10.1021/acsapm.9b01214

\section{General rights}

Copyright and moral rights for the publications made accessible in the public portal are retained by the authors and/or other copyright owners and it is a condition of accessing publications that users recognise and abide by the legal requirements associated with these rights.

- Users may download and print one copy of any publication from the public portal for the purpose of private study or research.

- You may not further distribute the material or use it for any profit-making activity or commercial gain

- You may freely distribute the URL identifying the publication in the public portal 


\title{
Performance of Polymeric Skin Adhesives during Perspiration
}

\author{
Daniel Hansen, Saeed Zajforoushan Moghaddam, Johannes Eiler, Kristoffer Hansen, \\ and Esben Thormann*
}

Cite This: ACS Appl. Polym. Mater. 2020, 2, 1535-1542

Read Online

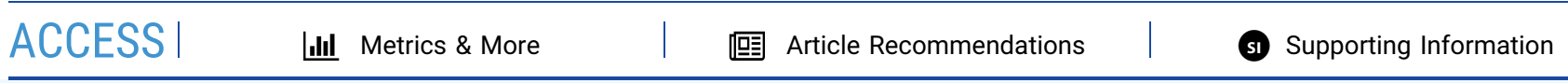

ABSTRACT: Skin adhesives are polymer materials used for attaching medical devices to the skin. Probing the performance of such adhesives is of great interest for rational material formulation. Here, we present a perspiration simulator, which includes a skin mimicking gelatin substrate with controlled roughness and the ability to perspire with a tunable sweat rate. The setup was used for probing peel adhesion of adhesives under realistic wear conditions. Adhesives with indistinguishable rheological properties but different ability to absorb artificial sweat were evaluated. The rheological
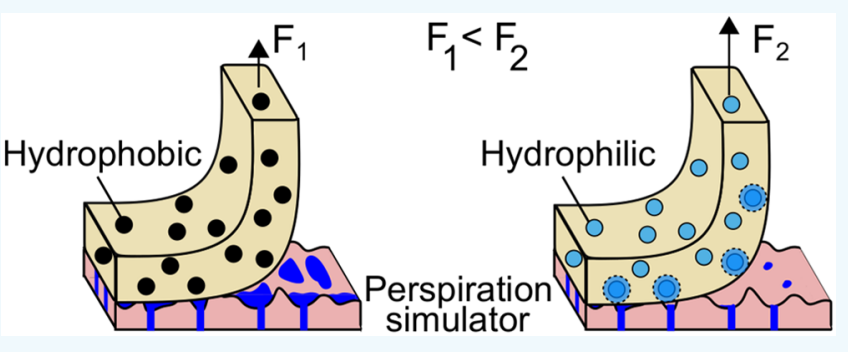
properties were fixed to decouple the bulk mechanical properties from events occurring at the substrate-adhesive interface. The effects of application pressure, dwell time, and perspiration were quantified for each adhesive formulation. Here, we found that sweat introduced at the substrate-adhesive interface restricts further bonding of the adhesives by limiting viscous flow. Water-absorbing skin adhesives were found to have significantly higher peel forces compared to nonabsorbing adhesives under sweating conditions where the adhesive could absorb the introduced sweat.

KEYWORDS: skin adhesives, perspiration simulator, peel adhesion, adhesive bonding, artificial skin

\section{INTRODUCTION}

Skin adhesives represent a class of polymer composites used for attaching medical devices, such as transdermal drugdelivery patches, ostomy bags, wound dressings, and wearable biosensors to the skin. ${ }^{1-9}$ Such adhesives must provide sufficient adhesion to ensure optimal functionality of the attached device, while being easy to remove without leaving residues or damaging the skin. ${ }^{8}$ These adhesives must also adhere to the skin during perspiration where sweat is released at the skin-adhesive interface. The presence of sweat can compromise adhesion by perturbing the nonspecific interactions between the adhesive and skin. ${ }^{9}$ Furthermore, prolonged exposure to sweat accumulated between the skin and adhesive causes skin maceration. ${ }^{10,11}$ To address this issue, modern formulations include hydrophilic fillers, known as hydrocolloids, in the sticky hydrophobic polymer matrix to remove bodily fluids from the skin-adhesive interface. ${ }^{12}$ However, rational formulation of skin adhesives still presents a challenge due to difficulties with probing adhesive performance during wear and balancing the antagonist requirements for adhesion.

Some clinical investigations, where adhesive performance during wear is evaluated, are available in the literature. Here, the adhesive performance is typically quantified through peel tests or by recording the wear time until the adhesive detaches from the skin of resting subjects. ${ }^{13,14}$ However, clinical studies are costly and require a large number of subjects to yield results of statistical significance due to large intra- and intersubject variations. ${ }^{15}$ Consequently, there is a growing demand to develop skin models for in vitro adhesive testing, which mimic relevant skin parameters, such as surface roughness, mechanical properties, hydration, and surface energy. ${ }^{16-19}$ A limited effort has been put into clinical investigation of the performance of skin adhesives specifically during perspiration. Difficulties associated with controlling, reproducing, and measuring human perspiration introduce sources of variations, which make the results obtained from in vivo sweat studies even more convoluted. ${ }^{20,21}$ This further emphasizes the need for a perspiration simulator for systematic adhesive testing.

The requirements for a perspiration simulator depend on the device functionality and materials tested. For biosensors the sweat composition and rate are of high importance, while the friction during perspiration is of interest for textiles. ${ }^{22-25}$ For adhesive testing the model needs to exert a pressure from the artificial sweat glands, possess a water contact angle similar to skin, and be tough enough to allow peeling adhesives from the skin. $^{26-28}$ Eiler et al. recently adapted a perspiration simulator, developed by Hou et al., ${ }^{29}$ to test the performance of skin adhesives under well-controlled perspiration conditions on a

Received: December 20, 2019

Accepted: March 4, 2020

Published: March 4, 2020 
polymer substrate mimicking the surface topography and water contact angle of skin. ${ }^{30}$ Rigid adhesives were chosen to specifically probe spreading of artificial sweat at the substrateadhesive interface without the influence of viscous flow of the adhesive. Adhesives with different abilities to absorb artificial sweat were evaluated by peel tests. Here, a delay in adhesive failure was observed for the adhesive absorbing the most artificial sweat. However, it is still unknown how perspiration influences the viscous flow of these adhesives and how the initial adhesive bonding affects the adhesive performance during perspiration for adhesives with different abilities to absorb sweat.

In this work, we present a new perspiration simulator having dimensions appropriate for conventional adhesive peel experiments. ${ }^{31}$ The setup provides the ability to control the artificial sweat rate, the hydrostatic pressure upon artificial sweat pore occlusion, sweat composition, and sweat pore density. The outermost layer of the substrate consists of a cross-linked gelatin film, which hydrates during water exposure mimicking human stratum corneum. ${ }^{32,33}$ The skin-like gelatin layer is coated on a rigid polyimide support with controlled roughness, hole size, and hole density. The coating process and crosslinking density of the gelatin were optimized to avoid blocking of the artificial sweat pores and cohesive fracturing of the gelatin during peel experiments. The model is used for systematic investigations of the peel adhesion of three different adhesive formulations under realistic perspiration conditions. The adhesive formulations are designed to have indistinguishable rheological properties yet significantly different abilities to absorb sweat. This enables us to decouple the bulk properties from the events occurring at the artificial skin-adhesive interface and separate the effects of water transport from the mechanical contributions to the peel adhesion. Each adhesive formulation is evaluated as a function of application pressure prior to artificial perspiration and different perspiration times to systematically investigate the link between adhesive bonding, amount of artificial sweat, water transport, and peel adhesion.

\section{MATERIALS AND METHODS}

2.1. Materials. Polyisobutylene was obtained from BASF (Germany), styrene-isoprene-styrene was obtained from Kraton (USA), sodium carboxymethyl cellulose (CMC) particles were obtained from Akzo Nobel (Netherlands), and potato starch was obtained from KMC (Denmark). A premix of styrene-isoprenestyrene and polyisobutylene (the adhesive matrix) was mixed for 45 min at $30 \mathrm{rpm}$ and $90{ }^{\circ} \mathrm{C}$ under vacuum in a Brabender mixer (Brabender GmbH \& Co. KG, Germany). The ratio between polyisobutylene and styrene-isoprene-styrene was kept at $4: 1$ by weight, and the total mass of each mix was $60 \mathrm{~g}$. The polyisobutylene provides the stickiness of the adhesives, while the styrene-isoprenestyrene is added for increasing the cohesive strength of the adhesives. Next, particles were mixed with the matrix to yield adhesives with a total particle volume fraction, $\phi_{\mathrm{p}}=0.277$. Three different adhesives were mixed: one containing only CMC fillers (hereafter CMC adhesive), one having half of the particle volume fraction CMC fillers and the other half starch fillers (hereafter CMC-Starch adhesive), and one only containing starch fillers (hereafter Starch adhesive). Particle densities were measured with an AccuPyc $1330 \mathrm{He}$ pycnometer from Micrometrics (USA). The densities of CMC and Starch were found to be $\rho_{\text {CMC }}=1.5809 \pm 0.0004 \mathrm{~g} \mathrm{~cm}^{-3}$ and $\rho_{\text {Starch }}=$ $1.5203 \pm 0.0009 \mathrm{~g} \mathrm{~cm}^{-3}$, respectively. Vacuum was applied after the particles were added to the matrix to minimize air entrapment, and the mix was processed for $45 \mathrm{~min}$ at $30 \mathrm{rpm}$ and $90^{\circ} \mathrm{C}$. After mixing, $20 \mathrm{~g}$ of material was pressed between two horizontal steel plates for 30 s under a load of 10 tons at $90^{\circ} \mathrm{C}$. The sample mixtures were pressed to a thickness of $950 \pm 25 \mu \mathrm{m}$. The thickness was subsequently measured by using a thickness gauge (Mitutoyo Europe GmbH, Germany). After pressing, the desired sample geometry was obtained by punching. The artificial sweat solution for the impedance measurements, gravimetric analysis, and artificial perspiration experiments was prepared from ultrapure water (arium pro, Satorius, Germany) and sodium chloride (Sigma-Aldrich, Denmark) at a concentration of $154 \mathrm{mM}$, which is within the range of the sodium chloride concentration of human sweat. ${ }^{34}$

2.2. Material Characterization. 2.2.1. Rheology. The rheological properties of each formulation were evaluated by shear rheology using a Discovery hybrid rheometer from TA Instruments (Denmark) with parallel plate geometry. Amplitude sweeps at $1 \mathrm{~Hz}$ were done for each formulation to identify the linear viscoelastic region. The amplitude sweeps are provided as Supporting Information (Figure S1). Frequency sweeps were subsequently performed on a new sample at fixed shear strain, $\hat{\gamma}=0.3 \%$. The frequency sweeps were done with frequencies ranging from $10^{2}$ to $10^{-3} \mathrm{~Hz}$ with five frequencies per decade. All measurements were performed in triplicates at $32{ }^{\circ} \mathrm{C}$, provided as Supporting Information (Figure S2).

2.2.2. Impedance Measurements. Impedance measurements were performed according to a method that we previously developed for probing water diffusion in polymer materials. ${ }^{35}$ The method utilizes a two-electrode system connected to an Autolab potentiostat (PGSTAT128N) with a frequency response analyzer module (FRA32M) and a multiplexer module (MUX.SCNR8) all obtained from Metrohm Nordic (Denmark). The adhesive was adhered to a stainless-steel plate (working electrode) and subsequently clamped to a glass cell. A graphite rod was used as both the counter and reference electrode. The adhesive was exposed to $154 \mathrm{mM} \mathrm{NaCl}$ solution. The adhesive area was chosen to be $9.6 \mathrm{~cm}^{2}$, which is much larger than the filler sizes, to average out sample inhomogeneities. An oscillating potential with an amplitude of $10 \mathrm{mV}$ was applied between the electrodes, and time scans were performed at $100 \mathrm{kHz}$. The temperature during the impedance measurements was adjusted to $32 \pm 1{ }^{\circ} \mathrm{C}$ with an incubator (KS 4000i control, IKA, Germany). The experiments were done in triplicates. The capacitance was obtained from the impedance by using an equivalent circuit model. Here, we model the adhesive as a (RC) circuit, i.e., a resistor and a capacitor in parallel. The resistor and capacitor represent bulk conductivity and the dielectric properties of the sample, respectively. The resistance of the hardware and electrolyte solution was neglected. The capacitance, $C$, of a (RC) circuit is readily obtained as

$$
C=-\frac{Z^{\prime \prime}}{\omega\left(Z^{\prime 2}+Z^{\prime \prime 2}\right)}
$$

where $\omega$ is the angular frequency of the potential, $Z^{\prime}$ is the real part of the impedance, and $Z^{\prime \prime}$ is the imaginary part of the impedance. The presented equivalent circuit model is valid until saline solution reaches the working electrode; thereafter, the electrical behavior of the working electrode may have a significant contribution. ${ }^{35}$

2.2.3. Gravimetric Analysis. Gravimetric analysis was performed on adhesive samples immersed in $154 \mathrm{mM} \mathrm{NaCl}$ solution using an analytical balance (XS105, Mettler Toledo, Denmark). The adhesives were $25 \times 25 \times 1 \mathrm{~mm}^{3}$ and adhered to a polystyrene plate during the experiment. The temperature of the solution was kept at $32{ }^{\circ} \mathrm{C}$. The mass of the adhesives was measured prior to immersion and after 10 , $30,45,60$, and $120 \mathrm{~min}$ of immersion. Each measurement was done five times.

2.3. Perspiration Simulator. The artificial skin is a multilayer substrate, where each layer holds a functionality. The skin includes a track-etched membrane (obtained from Sterlitech Cooperation, USA) of polycarbonate having a thickness $l_{\mathrm{m}}=12 \mu \mathrm{m}$, pore diameter $d_{\mathrm{m}}=$ $0.1 \mu \mathrm{m}$, and pore density $\alpha_{\mathrm{m}}=4 \mu \mathrm{m}^{-2}$. The track-etched membrane was laminated to the polyimide support by using a double-sided acrylic adhesive. The acrylic adhesive was applied to the polyimide support prior to drilling the artificial sweat pores to ensure the adhesive did not block the pores. The track-etched membrane was 
a

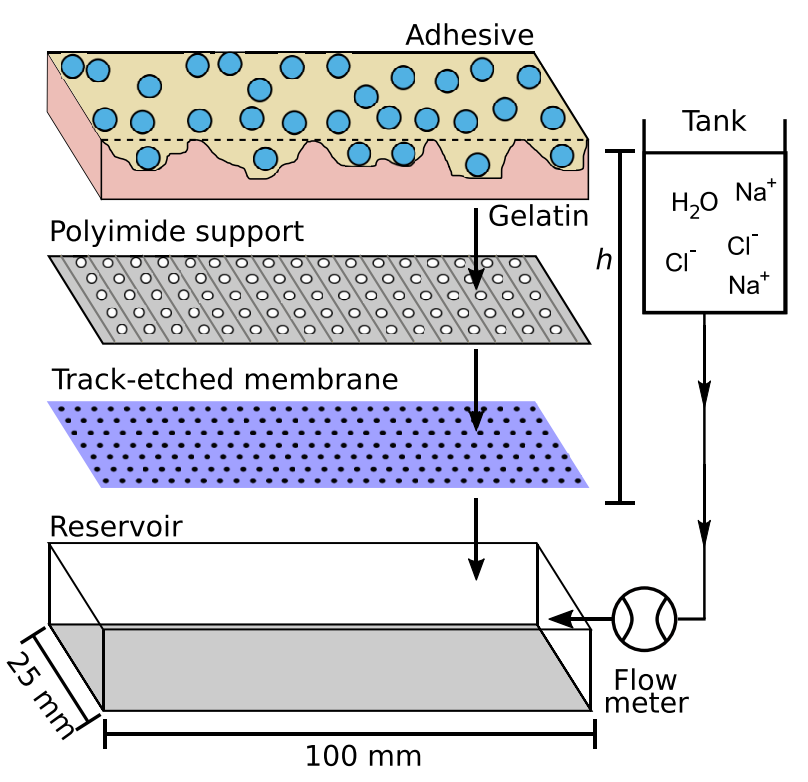

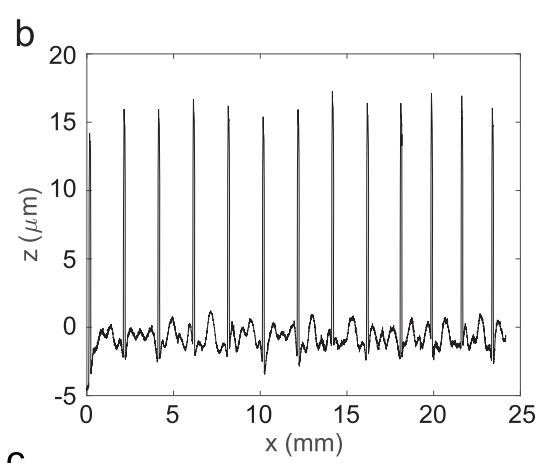

C

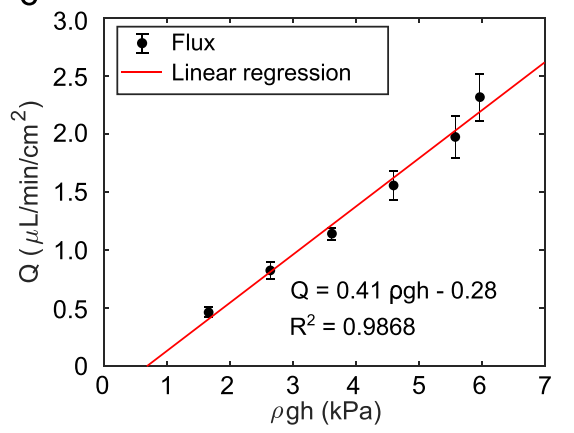

Figure 1. Schematic illustration of the artificial skin, which consists of a track-etched membrane and a polyimide substrate with micrometer-scale roughness coated with cross-linked gelatin. The artificial skin was clamped to a reservoir connected to a flow meter and a tank for artificial sweat with height, $h$, above the skin surface (a). A roughness line scan of the artificial skin obtained with a profilometer is shown in (b). The artificial perspiration rate is plotted as a function of pressure with a linear model fitted to the experimental data (c). The perspiration data represent averages and standard deviations of three different artificial skins.

carefully laminated to the polyimide substrate, and the skin was left for $24 \mathrm{~h}$ with a pressure of $250 \mathrm{~Pa}$ to allow the adhesive to bond with the track-etched membrane. The polyimide support has a well-defined micrometer-scale roughness and holes having a diameter $d_{\mathrm{s}}=250 \mu \mathrm{m}$ and hole density $\alpha_{\mathrm{s}}=100 \mathrm{~cm}^{-2}$. The holes in the polyimide support are supposed to mimic the sweat pores. The track-etched membrane provides high flow resistance, which yields a pressure drop much larger than the differences in Laplace pressure of the holes in the polyimide support, which ensures homogeneous sweating throughout the substrate. ${ }^{29}$ The polyimide support gives mechanical stability and large scale roughness. The polyimide support was coated with gelatin (Figure 1a) through a dip-coating procedure (details are provided in the Supporting Information, section S2). For less complex adhesion results, the gelatin was highly cross-linked to avoid cohesive failure and elastic deformation of the artificial skin. This is different from human skin, which is elastic and typically allows for removal of superficial skin cells. Gelatin is derived from collagen, which is a natural component of human skin. ${ }^{36}$ In the Supporting Information, we furthermore provide quartz crystal microbalance with dissipation measurements to confirm that the gelatin film is hydrated through water exposure (Figure S3) and atomic force microscopy images to illustrate the nanoscale roughness of the gelatin (Figure S4). The thickness of the gelatin layer was estimated to be a few micrometers through atomic force microscopy and gravimetric measurements (Figure S5). The water contact angle was measured to $77.5 \pm 0.8^{\circ}$, which is similar to reported values measured on human skin. ${ }^{37,38}$ The experimental details and the data of the water contact angle are provided in the Supporting Information (Figure S6). The mean surface roughness of the artificial skin was measured by using a profilometer (SJ-410 surface roughness tester, Mitutoyo, Germany). A line scan is shown in Figure $1 \mathrm{~b}$ where the surface height, $z$, is plotted as a function of distance, $x$. The average roughness parameter was $R_{\mathrm{a}}$ $=8.4 \pm 4.5 \mu \mathrm{m}$, which is on the same order of magnitude as reported literature values for human skin. ${ }^{39,40}$

The artificial skin was clamped to a reservoir, which was connected a microfluidic flow sensor and a tank supplying the artificial sweat solution (Figure 1a). The flow sensor was connected to a microfluidic flow reader both obtained from Elveflow (France). The flow sensor allowed to measure the artificial sweating rate, which is a function of the tank height, $h$. Flow rates were measured as a function of pressure. The data are presented in Figure 1c. Here, a linear flow rate-pressure relation was found, which was expected from Poiseuille's law:

$$
Q_{\mathrm{n}}=\frac{\pi^{2} d_{\mathrm{m}}{ }^{4} d_{\mathrm{s}} \alpha_{m}}{512 \eta l_{\mathrm{m}}}\left(\rho g h d_{s}-4 \gamma \cos (\theta)\right)
$$

where $Q_{n}$ is the flow in a single artificial sweat pore, $\theta$ is the contact angle of the substrate with the artificial sweat, $g$ is the gravitational constant, and $\eta, \gamma$, and $\rho$ are the viscosity, surface tension, and density of the artificial sweat. The remaining parameters are described above. Equation 2 was derived under the assumption that the resistance in the polyimide support is negligible compared to the resistance of the track-etched membrane. The final expression is a linear relationship between the flow rate in an artificial sweat pore and the pressure, where the proportionality constant is the inverse resistance of the sweat pore and the intercept with the $x$-axis is the Laplace pressure in a pore, $\Delta p_{\mathrm{L}}=\frac{4 \gamma \cos \theta}{d_{\mathrm{s}}} .29$

2.3.1. Peel Test. The adhesive properties were evaluated by using an Instron 5943 Universal testing system with a $50 \mathrm{~N}$ load cell (Instron, Sweden). The dimensions of the adhesives were $100 \times 25 \times$ $1 \mathrm{~mm}^{3}$. The adhesives were applied to the artificial skin with either a pressure of 1680 or $4320 \mathrm{~Pa}$ for $60 \mathrm{~s}$ and were subsequently exposed to artificial perspiration or left to dwell at dry conditions. The adhesives were peeled with constant displacement, $304 \mathrm{~mm} / \mathrm{min}$, at an angle of $90^{\circ}$ by using a backing tape (4124, Tesa, Denmark). The force required to peel the adhesive from the substrate was recorded as a function of displacement. Recorded forces were averaged over displacements from 20 to $80 \mathrm{~mm}$ for each measurement, which was done in triplicates. We note that the artificial skins were not reused for multiple peel experiments and the skins were used within 2 weeks after preparation. 


\section{RESULTS AND DISCUSSION}

3.1. Characterization of Adhesive Formulations. The rheological properties of the adhesive formulations were characterized by shear rheology in the linear viscoelastic region, which was determined through amplitude sweeps (see Supporting Information, Figure S1). Frequency sweeps were performed at frequencies ranging from $10^{2}$ to $10^{-3} \mathrm{~Hz}$ with five frequencies per decade at a fixed shear strain, $\hat{\gamma}=0.3 \%$. The storage modulus, $G^{\prime}$, the loss modulus, $G^{\prime \prime}$, and the ratio between the loss and storage modulus, $\tan (\delta)=\frac{G^{\prime \prime}}{G^{\prime}}$, are displayed in Figure 2 as a function of the frequency.

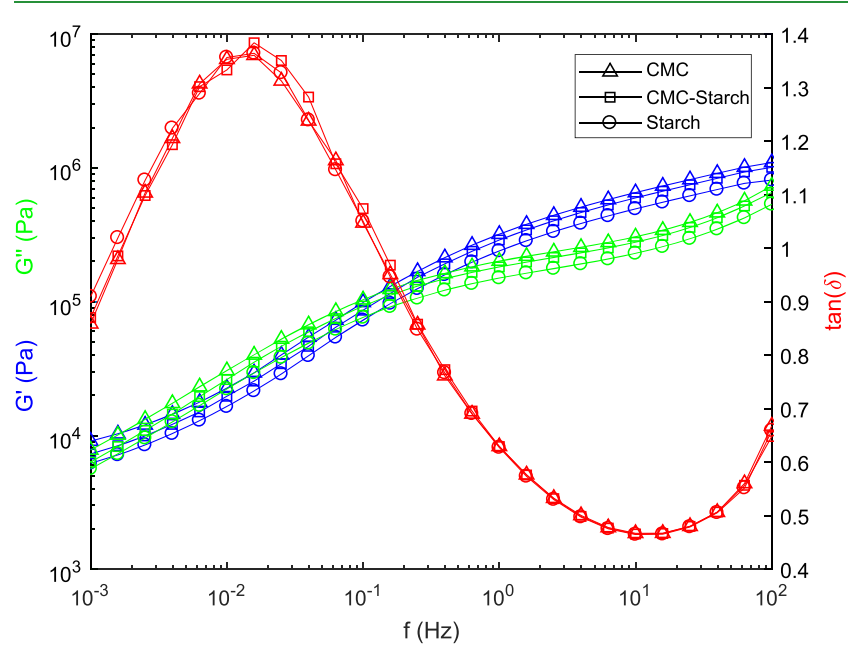

Figure 2. Storage modulus, $G^{\prime}$, loss modulus, $G^{\prime \prime}$, and $\tan (\delta)$ plotted as a function of deformation frequency, $f$, for the CMC $(\triangle)$, CMCStarch $(\square)$, and Starch (O) adhesives.

The rheological measurements were done in triplicates (see Figure S2) to ensure that the data in Figure 2 represent the general rheological properties. Variations within each adhesive formulation were similar to variations between formulations implying the three adhesive formulations have indistinguishable rheological properties in the measured frequency range. The different adhesives are thus expected to flow similarly when applied to the substrate.

Next, the water transport in the adhesives was investigated by using impedance measurements. The measurements were done with an alternating potential, with an amplitude of 10 $\mathrm{mV}$, oscillating at fixed frequency, $f=100 \mathrm{kHz}$, to increase the sensitivity to dielectric changes. The results are displayed in Figure 3.
The impedance and phase angle are plotted as a function of time for the Starch adhesives in Figure 3a. Here, the phase angle is constant at $\phi=-90^{\circ}$, indicating pure capacitive behavior. The impedance is constant for at least $1400 \mathrm{~min}$ $(\sim 23 \mathrm{~h})$, which means no water transport occurs in the Starch adhesive within this time. The CMC-Starch adhesives (Figure 3b) initially exhibit pure capacitive properties, $-\phi=90^{\circ}$, followed by the occurrence of bulk conductivity, indicated by a rapid drop in the phase angle. The conductivity occurs when the $\mathrm{NaCl}$ solution has penetrated through the adhesive and reached the working electrode. The rapid drop in phase angle is detected after $141 \pm 46 \mathrm{~min}$ where the large standard deviation, i.e., large intersample variation, implies poor connectivity of the water transporting CMC particles, which makes the water transport results sensitive to small variations in the particle dispersion between adhesive samples. Finally, the CMC adhesive (Figure 3c) shows the occurrence of full penetration after $107 \pm 3$ min of immersion, where the small standard deviation indicates continuous transport and good $\mathrm{CMC}$-particle connectivity, which is in agreement with the higher CMC-particle content.

The sweat uptake is further evaluated through the change in temporal capacitance relative to the dry capacitance, $\frac{C_{t}-C_{0}}{C_{t}}$, where $C_{t}$ and $C_{0}$ are the capacitance at a given time, $t$, and the dry capacitance, respectively. The capacitance is calculated by using eq 1 and the results for the CMC and CMC-Starch adhesives are shown in Figure 4. Here, the increase in the capacitance for the CMC adhesive is larger compared to the CMC-Starch adhesive, which implies that the water uptake in the CMC adhesives are higher than the CMC-Starch adhesives. Such behavior is expected from the respective chemistries of CMC and starch.

The sweat-absorbing properties of each adhesive formulation were also evaluated through gravimetric analysis of the composites immersed in artificial sweat (154 $\mathrm{mM} \mathrm{NaCl}$ ) solution. The absorbing properties were examined for up to $120 \mathrm{~min}$, which is longer than the longest time in the in vitro perspiration experiments. The results are presented in Figure 5, where the mass difference per adhesive area is plotted as a function of time. Each data point in Figure 5 represents an average of five measurements with error bars representing their respective standard deviations.

The CMC adhesives demonstrate a continuous uptake over the entire measured time range, while the CMC-Starch adhesives show an initial uptake within $10 \mathrm{~min}$ followed by a plateau indicating poor CMC particle connectivity. Lastly, the Starch adhesives show no water absorption, which again
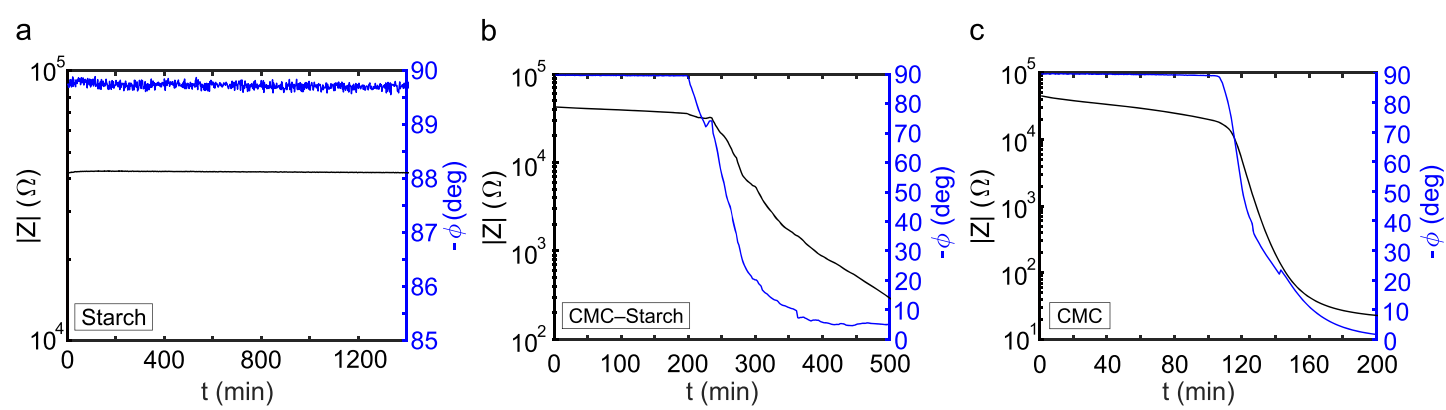

Figure 3. Impedance as a function of immersion time of Starch (a), CMC-Starch (b), and CMC (c) adhesives. The measurements were done using $154 \mathrm{mM} \mathrm{NaCl}$ solution at fixed frequency, $f=100 \mathrm{kHz}$. 


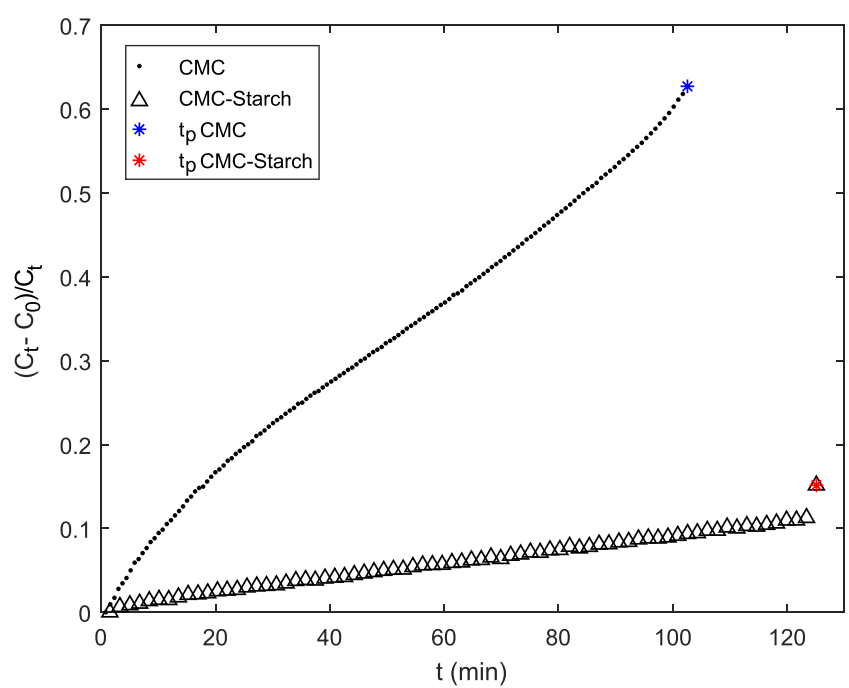

Figure 4. Difference in capacitance relative to the dry capacitance as a function of immersion time for the CMC $(\bullet)$ and CMC-Starch $(\triangle)$ adhesives. The asterisks $(*)$ represent the characteristic penetration times, $t_{\mathrm{p} .}$.

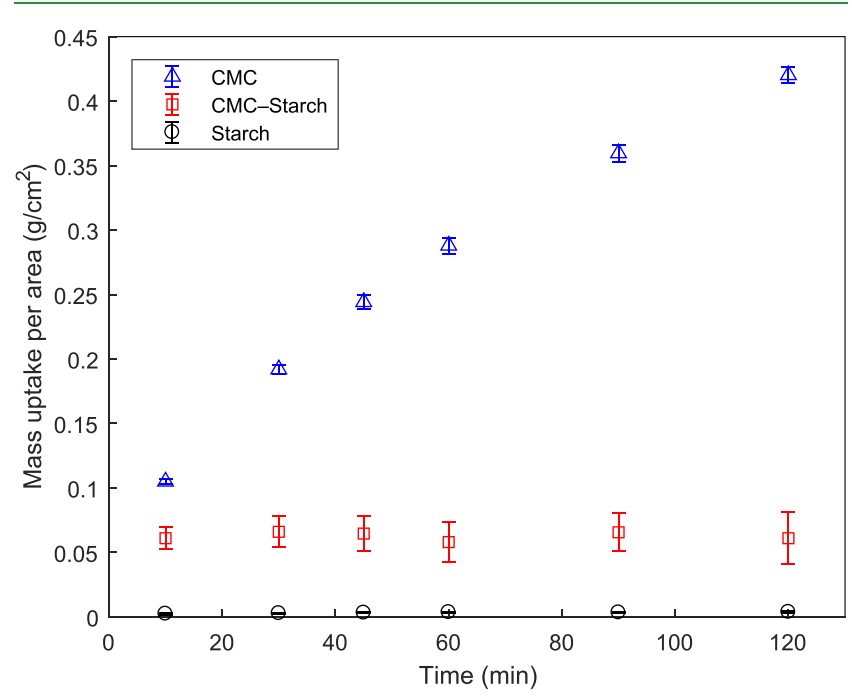

Figure 5. Mass uptake as a function of time for the CMC $(\triangle)$, CMC-Starch $(\square)$, and Starch $(O)$ adhesives immersed in $154 \mathrm{mM}$ $\mathrm{NaCl}$ solution.

confirms that starch does not absorb sweat under these conditions. These results are in agreement with the impedance measurements. The results in Figure 5 represent the absolute uptake per area when the entire adhesive surface area is exposed to artificial sweat. However, in the situation where the adhesive is attached to the perspiration simulator, the adhesive is expected to absorb locally around the artificial sweat pores. The actual uptake in a perspiration situation is therefore expected to be lower compared to the immersion tests.

3.2. Adhesive Performance during Artificial Perspiration. The aim of the work presented here is to demonstrate the potential of the perspiration simulator and obtain general knowledge of adhesive performance under realistic perspiration conditions. The adhesive performance was evaluated by peeling the adhesives, with an angle of $90^{\circ}$, directly from the perspiration simulator. The peel results are shown in Figures $6 \mathrm{a}$ and $6 \mathrm{~b}$ where the horizontal bars represent groups with equal means according to a two sample $t$ test with $95 \%$ confidence level. We divide the discussion of the peel results in two parts: adhesion under dry conditions and adhesion after artificial perspiration.

3.2.1. Adhesion under Dry Conditions. First, the adhesives were peeled from the artificial skin without introducing artificial sweat. This was done to study the effect of the application pressure and dwell time. Both dwell time and application pressure will have an effect on the substrateadhesive contact area due to the viscoelastic behavior of the adhesives. To obtain two different initial substrate-adhesive contact areas, we vary the application pressure. Here, each adhesive formulation was applied with respective pressures of $4320 \mathrm{~Pa}$ (Figure 6a) and $1680 \mathrm{~Pa}$ (Figure 6b) for $60 \mathrm{~s}$. Adhesives peeled immediately after application $(0 \mathrm{~min}$ after application) showed no significant differences between formulations as expected from their rheological properties and similar chemistries. However, there is a clear effect of the application pressure since the average peel forces were approximately 3 and $20 \mathrm{~N}$ for the adhesives applied with 1680 and $4320 \mathrm{~Pa}$, respectively. The increase in peel forces for increasing application pressure is a consequence of increasing viscous flow of the adhesive, which results in a larger initial substrate-adhesive contact area.

Adhesives were also peeled $20 \mathrm{~min}$ after application to investigate the effect of dwell time on peel adhesion. Here, the adhesives generally show increments in peel forces indicating the evolution of the substrate-adhesive contact area over time through viscous flow as illustrated in Figure 6c. After $20 \mathrm{~min}$ dwell time, differences were found between the CMC adhesive and the two other adhesives. Mixed failure modes were generally observed for peel forces larger than $15 \mathrm{~N}$. During mixed failure, both adhesive and cohesive failure occur, which suggests that the adhesion to the substrate was stronger locally than the cohesion of the adhesives. It also demonstrates that the skin-mimicking gelatin does not delaminate or fracture during peel. The structural integrity of the gelatin makes the peel results less convoluted compared to real human skin where adhesives peeled from the skin typically remove a superficial layer of stratum corneum. ${ }^{41}$ We speculate that the differences after $20 \mathrm{~min}$ are attributed to differences in nonlinear rheological properties between the CMC adhesive and the other two adhesive formulations.

3.2.2. Adhesion after Artificial Perspiration. Next, the peel adhesion of the adhesives, applied under the same conditions as above (4320 or $1680 \mathrm{~Pa}$ for $60 \mathrm{~s}$ ), was evaluated after 20 min of artificial perspiration with an artificial sweat rate corresponding to $1.6 \pm 0.2 \mu \mathrm{L} \mathrm{min}^{-1} \mathrm{~cm}^{-2}$ for uncovered skin. Because of partial occlusion of the artificial skin, the perspiration rates reduced to approximately $1.0 \pm 0.1 \mu \mathrm{L} /$ $\left(\min \mathrm{cm}^{2}\right.$ ) in all cases (see Figure S7). We note that even though water is an effective plasticizer, ${ }^{42-44}$ the changes in peel forces during perspiration are mainly expected to be a result of events occurring at the substrate-adhesive interface. This assumption is based on a conservative estimate of the changes in the bulk mechanical properties of the adhesives during perspiration, which is provided in the Supporting Information (section S4).

Peel forces measured after 20 min of artificial perspiration were lower compared to $20 \mathrm{~min}$ of dry dwell after application for all adhesive formulations and both application pressures. This revealed that the viscous flow of the adhesives is restricted due to the artificial sweat introduced at the substrate-adhesive interface as illustrated in Figure 6d. The Starch adhesive shows 


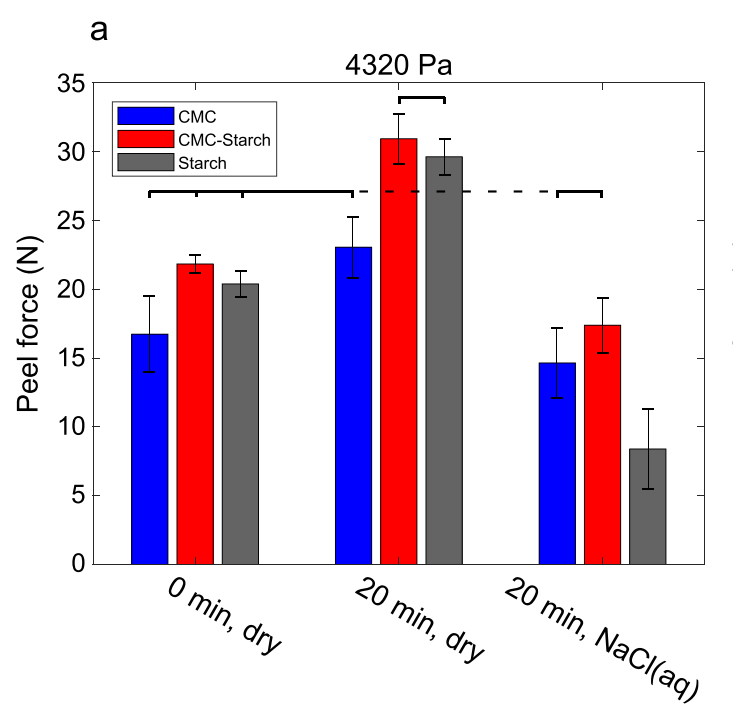

C

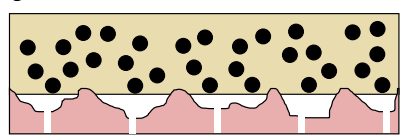

Dry dwell $\downarrow$

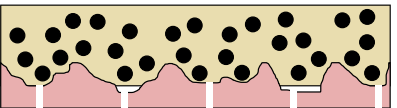

b

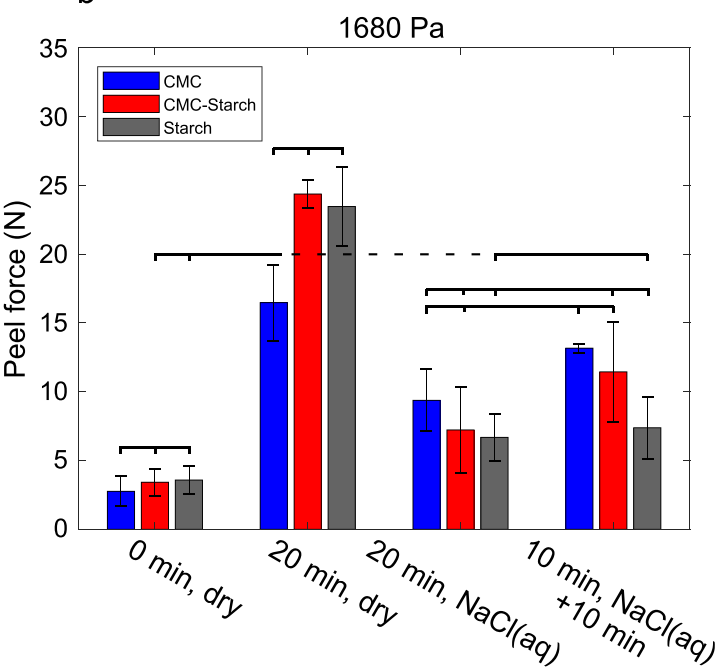

e
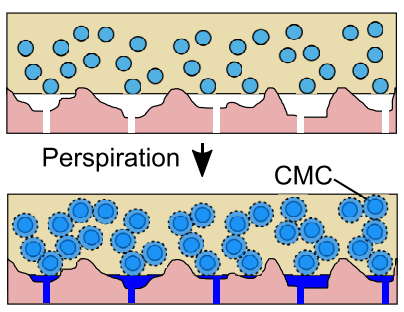

Figure 6. Average peel forces recorded of the CMC, CMC-Starch, and Starch adhesives peeled from the perspiration simulator. The adhesives were applied with application pressures of $4320 \mathrm{~Pa} \mathrm{(a)} \mathrm{and} 1680 \mathrm{~Pa}(\mathrm{~b})$ and peeled immediately after application and after 20 min without and with exposure to perspiration. The horizontal bars indicate groups with equal means (two sample $t$ test, $95 \%$ confidence level). Schematic illustrations of the substrate-adhesive interface region, which are based on the peel results, depicting dry dwell (c), perspiration with a Starch adhesive (d), and CMC adhesive (e).

significantly lower peel force compared to the initial value $(0$ min) at high application pressure (Figure 6a). This suggests that some of the initial substrate-adhesive contact area is compromised due to the pressure exerted from the artificial sweat glands $(\sim 4.5 \mathrm{kPa})$, which we speculate is a consequence of the Starch adhesive's occlusive nature. The CMC and CMC-Starch adhesives are less occlusive through their water absorption and consequently do not show this significant drop in peel force. For the low application pressure (Figure 6b), the peel forces after $20 \mathrm{~min}$ do not decrease below the initial ( 0 min) peel forces, which indicates that the initial substrateadhesive contact area remains throughout the perspiration process. Here, the adhesives are less occlusive due to a smaller substrate-adhesive contact area, which will result in a lower overall pressure build up at the interface. However, artificial sweat spreading at the substrate surface restricts further adhesive bonding with the substrate. After $20 \mathrm{~min}$ of perspiration, no significant differences are detected between formulations.

Next, the perspiration time was decreased to $10 \mathrm{~min}$ to introduce an overall lower volume of artificial sweat at the interface. After $10 \mathrm{~min}$ of perspiration, the adhesives were left on the artificial skin for an additional $10 \mathrm{~min}$ without perspiration and was subsequently peeled off the artificial skin (Figure 6b). The Starch adhesive shows no change in peel force compared to $20 \mathrm{~min}$ of artificial perspiration. This suggests that sufficient artificial sweat accumulates at the interface during $10 \mathrm{~min}$ to prohibit further adhesive bonding due to lack of sweat absorption. However, the CMC adhesive shows an increase in peel force and now performs significantly better compared to the Starch adhesive. This indicates that having hydrophilic fillers near the skin-adhesive interface to absorb sweat maintains the adhesive properties during perspiration. The hydrophilic CMC fillers absorb sweat at the substrate-adhesive interface, which allows the adhesive to flow and bond to the substrate and consequently increase the substrate-adhesive contact area over time (Figure 6e). The CMC-Starch adhesive seem to exhibit peel forces in between the CMC and Starch adhesive as expected from its water absorption capacity, which also is in between the two other adhesives.

\section{CONCLUSION}

A new perspiration simulator for evaluating the performance of skin adhesives during realistic wear conditions was successfully developed. The perspiration simulator has a gelatin skin mimicking substrate and the ability to sweat with a controlled sweat rate. Three skin adhesive formulations having indistinguishable rheological properties, but different abilities to absorb the artificial sweat were evaluated by measuring the peel adhesion directly from the perspiration simulator. Artificial sweat introduced at the artificial skin-adhesive interface proved to restrict viscous flow of the adhesives and consequently reduced the adhesive's ability to bond to the substrate. However, highly water-absorbing adhesives were found to bond under perspiration conditions depending on the total amount of artificial sweat introduced. Here, the absorbing 
adhesive showed a significant increase in peel adhesion compared to non-sweat-absorbing adhesives.

\section{ASSOCIATED CONTENT}

\section{SI Supporting Information}

The Supporting Information is available free of charge at https://pubs.acs.org/doi/10.1021/acsapm.9b01214.

Additional rheological measurements, description of the gelatin coating process, characterization of the gelatin, and artificial perspiration rates (PDF)

\section{AUTHOR INFORMATION}

\section{Corresponding Author}

Esben Thormann - Department of Chemistry, Technical University of Denmark, 2800 Kgs. Lyngby, Denmark; ○ orcid.org/0000-0002-2364-3493; Email: esth@ kemi.dtu.dk

\section{Authors}

Daniel Hansen - Department of Chemistry, Technical University of Denmark, 2800 Kgs. Lyngby, Denmark; Coloplast A/S, 3050 Humlebrek, Denmark; 이이.org/0000-00016834-9450

Saeed Zajforoushan Moghaddam - Department of Chemistry, Technical University of Denmark, 2800 Kgs. Lyngby, Denmark; 이이.org/0000-0002-6536-7490

Johannes Eiler - Department of Chemistry, Technical University of Denmark, 2800 Kgs. Lyngby, Denmark; Coloplast A/S, 3050 Humlebak, Denmark

Kristoffer Hansen - Coloplast A/S, 3050 Humlebak, Denmark

Complete contact information is available at:

https://pubs.acs.org/10.1021/acsapm.9b01214

\section{Notes}

The authors declare no competing financial interest.

\section{ACKNOWLEDGMENTS}

Coloplast A/S and Innovation Fund Denmark is gratefully acknowledged for financial support through the Grand Solutions Project \#6151-00007B. The authors thank Bahar Bingöl, Aamir Shabbir, and Irakli Javakhishvili for discussions.

\section{REFERENCES}

(1) Prausnitz, M. R.; Langer, R. Transdermal Drug Delivery. Nat. Biotechnol. 2008, 26, 1261.

(2) Lyon, C.; Smith, A.; Griffiths, C.; Beck, M. The Spectrum of Skin Disorders in Abdominal Stoma Patients. Br. J. Dermatol. 2000, 143, $1248-1260$.

(3) Windmiller, J. R.; Wang, J. Wearable Electrochemical Sensors and Biosensors: A Review. Electroanalysis 2013, 25, 29-46.

(4) Venkatraman, S.; Gale, R. Skin Adhesives and Skin Adhesion: 1. Transdermal Drug Delivery Systems. Biomaterials 1998, 19, 11191136.

(5) Gu, Z.; Wan, X.; Lou, Z.; Zhang, F.; Shi, L.; Li, S.; Dai, B.; Shen, G.; Wang, S. Skin Adhesives with Controlled Adhesion by Polymer Chain Mobility. ACS Appl. Mater. Interfaces 2019, 11, 1496-1502.

(6) Kim, D. W.; Baik, S.; Min, H.; Chun, S.; Lee, H. J.; Kim, K. H.; Lee, J. Y.; Pang, C. Highly Permeable Skin Patch with Conductive Hierarchical Architectures Inspired by Amphibians and Octopi for Omnidirectionally Enhanced Wet Adhesion. Adv. Funct. Mater. 2019, 29, 1807614.

(7) Wan, X.; Gu, Z.; Zhang, F.; Hao, D.; Liu, X.; Dai, B.; Song, Y.; Wang, S. Asymmetric Janus Adhesive Tape Prepared by Interfacial
Hydrosilylation for Wet/Dry Amphibious Adhesion. NPG Asia Mater. 2019, 11, 1-9.

(8) Chivers, R. A. Easy Removal of Pressure Sensitive Adhesives for Skin Applications. Int. J. Adhes. Adhes. 2001, 21, 381-388.

(9) Yuk, H.; Varela, C. E.; Nabzdyk, C. S.; Mao, X.; Padera, R. F.; Roche, E. T.; Zhao, X. Dry Double-Sided Tape for Adhesion of Wet Tissues and Devices. Nature 2019, 575, 169-174.

(10) Wokovich, A. M.; Prodduturi, S.; Doub, W. H.; Hussain, A. S.; Buhse, L. F. Transdermal Drug Delivery System (TDDS) Adhesion as a Critical Safety, Efficacy and Quality Attribute. Eur. J. Pharm. Biopharm. 2006, 64, 1-8.

(11) Cutting, K. F.; White, R. J. Maceration of the Skin and Wound Bed 1: Its Nature and Causes. J. Wound Care 2002, 11, 275-278.

(12) Creton, C.; Ciccotti, M. Fracture and Adhesion of Soft Materials: A Review. Rep. Prog. Phys. 2016, 79, 046601.

(13) Karwoski, A.; Plaut, R. Experiments on Peeling Adhesive Tapes from Human Forearms. Skin Res. Technol. 2004, 10, 271-277.

(14) Tokumura, F.; Homma, T.; Tomiya, T.; Kobayashi, Y.; Matsuda, T. Properties of Pressure-Sensitive Adhesive Tapes with Soft Adhesives to Human Skin and their Mechanism. Skin Res. Technol. 2007, 13, 211-216.

(15) Cunningham, D. D.; Lowery, M. G. Moisture Vapor Transport Channels for the Improved Attachment of a Medical Device to the Human Body. Biomed. Microdevices 2004, 6, 149-154.

(16) Renvoise, J.; Burlot, D.; Marin, G.; Derail, C. Adherence Performances of Pressure Sensitive Adhesives on a Model Viscoelastic Synthetic Film: A Tool for the Understanding of Adhesion on the Human Skin. Int. J. Pharm. 2009, 368, 83-88.

(17) Charkoudian, J. C. A Model Skin Surface for Testing Adhesion to Skin, 1988.

(18) Wokovich, A. M.; Brown, S. A.; McMaster, F. J.; Doub, W. H.; Cai, B.; Sadrieh, N.; Chen, M. L.; Machado, S.; Shen, M.; Buhse, L. F. Evaluation of Substrates for $90^{\circ}$ Peel Adhesion-A Collaborative Study. I. Medical Tapes. J. Biomed. Mater. Res., Part B 2008, 87, 105113.

(19) Baker, L. B. Sweating Rate and Sweat Sodium Concentration in Athletes: A Review of Methodology and Intra/Interindividual Variability. Sports Med. 2017, 47, 111-128.

(20) Smith, C. J.; Havenith, G. Body Mapping of Sweating Patterns in Athletes: A Sex Comparison. Med. Sci. Sports Exercise 2012, 44, 2350-2361.

(21) Taylor, N. A.; Machado-Moreira, C. A. Regional Variations in Transepidermal Water Loss, Eccrine Sweat Gland Density, Sweat Secretion Rates and Electrolyte Composition in Resting and Exercising Humans. Extreme Physiol. Med. 2013, 2, 4.

(22) Cho, E.; Mohammadifar, M.; Choi, S. A Self-Powered Sensor Patch for Glucose Monitoring in Sweat. 2017 IEEE 30th International Conference on Micro Electro Mechanical Systems (MEMS), 2017; pp $366-369$.

(23) Weber, J.; Kumar, A.; Kumar, A.; Bhansali, S. Novel Lactate and $\mathrm{pH}$ Biosensor for Skin and Sweat Analysis Based on Single Walled Carbon Nanotubes. Sens. Actuators, B 2006, 117, 308-313.

(24) Derler, S.; Rao, A.; Ballistreri, P.; Huber, R.; Scheel-Sailer, A.; Rossi, R. Medical Textiles with Low Friction for Decubitus Prevention. Tribol. Int. 2012, 46, 208-214.

(25) Bertaux, E.; Derler, S.; Rossi, R. M.; Zeng, X.; Koehl, L.; Ventenat, V. Textile, Physiological, and Sensorial Parameters in Sock Comfort. Text. Res. J. 2010, 80, 1803-1810.

(26) Heikenfeld, J. Bioanalytical Devices: Technological Leap for Sweat Sensing. Nature 2016, 529, 475.

(27) Gerhardt, L.-C.; Schiller, A.; Müller, B.; Spencer, N.; Derler, S. Fabrication, Characterisation and Tribological Investigation of Artificial Skin Surface Lipid Films. Tribol. Lett. 2009, 34, 81.

(28) Gal, A.; Nussinovitch, A. Plasticizers in the Manufacture of Novel Skin-Bioadhesive Patches. Int. J. Pharm. 2009, 370, 103-109.

(29) Hou, L.; Hagen, J.; Wang, X.; Papautsky, I.; Naik, R.; KelleyLoughnane, N.; Heikenfeld, J. Artificial Microfluidic Skin for In Vitro Perspiration Simulation and Testing. Lab Chip 2013, 13, 1868-1875. 
(30) Eiler, J.; Hansen, D.; Bingöl, B.; Hansen, K.; Heikenfeld, J.; Thormann, E. In vitro Evaluation of Skin Adhesives during Perspiration. Int. J. Adhes. Adhes. 2020, 99, 102574.

(31) Gu, Z.; Li, S.; Zhang, F.; Wang, S. Understanding Surface Adhesion in Nature: A Peeling Model. Adv. Sci. 2016, 3, 1500327.

(32) Lee, S. B.; Jeon, H. W.; Lee, Y. W.; Lee, Y. M.; Song, K. W.; Park, M. H.; Nam, Y. S.; Ahn, H. C. Bio-Artificial Skin Composed of Gelatin and $(1 \rightarrow 3),(1 \rightarrow 6)-\beta$-Glucan. Biomaterials 2003, 24 , 2503-2511.

(33) Parvez, S.; Rahman, M. M.; Khan, M. A.; Khan, M. A. H.; Islam, J. M.; Ahmed, M.; Rahman, M. F.; Ahmed, B. Preparation and Characterization of Artificial Skin using Chitosan and Gelatin Composites for Potential Biomedical Application. Polym. Bull. 2012, 69, 715-731.

(34) Cage, G. W.; Dobson, R. L. Sodium Secretion and Reabsorption in the Human Eccrine Sweat Gland. J. Clin. Invest. 1965, 44, 1270-1276.

(35) Hansen, D.; Brewer, J. R.; Eiler, J.; Komjani, N. M.; Hansen, K.; Thormann, E. Water Diffusion in Polymer Composites Probed by Impedance Spectroscopy and Time-Resolved Chemical Imaging. ACS Appl. Polym. Mater. 2020, 2, 837-845.

(36) Dabrowska, A.; Rotaru, G.M.; Spano, F.; Affolter, C..; Fortunato, G.; Lehmann, S.; Derler, S.; Spencer, N.D.; Rossi, R.M. A Water-Responsive, Gelatine-Based Human Skin Model. Tribol. Int. 2017, 113, 316-322.

(37) Mavon, A.; Zahouani, H.; Redoules, D.; Agache, P.; Gall, Y.; Humbert, P. Sebum and Stratum Corneum Lipids Increase Human Skin Surface Free Energy as Determined from Contact Angle Measurements: A Study on Two Anatomical Sites. Colloids Surf., B 1997, 8, 147-155.

(38) Ginn, M.; Noyes, C.; Jungermann, E. The Contact Angle of Water on Viable Human Skin. J. Colloid Interface Sci. 1968, 26, 146151.

(39) Cook, T. H.; Craft, T. J.; Brunelle, R. L.; Norris, F.; Griffin, W. A. Quantification of the Skin's Topography by Skin Profilometry. Int. J. Cosmet. Sci. 1982, 4, 195-205.

(40) Lagarde, J.; Rouvrais, C.; Black, D. Topography and Anisotropy of the Skin Surface with Ageing. Skin Res. Technol. 2005, 11, 110119.

(41) Cutting, K. Impact of Adhesive Surgical Tape and Wound Dressings on the Skin, with Reference to Skin Stripping. J. Wound Care 2008, 17, 157-162.

(42) Gontard, N.; Guilbert, S.; CUQ J.-L. Water and Glycerol as Plasticizers Affect Mechanical and Water Vapor Barrier Properties of an Edible Wheat Gluten Film. J. Food Sci. 1993, 58, 206-211.

(43) Long, F.; Thompson, L. Water Induced Acceleration of the Diffusion of Organic Vapors in Polymers. J. Polym. Sci. 1954, 14, 321-327.

(44) Rathna, G.; Rao, D. M.; Chatterji, P. Water-Induced Plasticization of Solution Cross-Linked Hydrogel Networks: Energetics and Mechanism. Macromolecules 1994, 27, 7920-7922. 